\title{
ROTATION AND MACROTURBULENCE IN SUPERGIANT STARS
}

\author{
JEFFREY D. ROSENDHAL \\ Washburn Observatory, University of Wisconsin, Madison, Wis., U.S.A.
}

\begin{abstract}
A statistical investigation has been made of rotation and macroturbulence in early type Ia and Iab supergiants. The principal results are: (1) At all spectral types in the range B0-A5 both rotation and macroturbulence contribute to the observed line broadening. In the early B stars rotation is as important a broadening agent as large-scale mass motion. In the middle B and A stars turbulence dominates but there still is an appreciable contribution from rotation. (2) In spite of the fact that the stars observed seem to be losing mass, there is no strong evidence for significant angular momentum loss.
\end{abstract}

\section{Introduction}

Recent observational studies (Oke and Greenstein, 1954; Sandage, 1955; Abt, 1957; Faber and Danziger, 1969) of the compatibility of the observed axial rotation of stars and currently accepted theories of stellar evolution have suggested that for stars of Iuminosity classes IV, III and II earlier than spectral type G0 angular momentum is conserved during evolution. In the later spectral types of all luminosity classes the rotation is very low, and it is tempting to correlate this fact with the development of extensive outer convective zones and subsequent loss of angular momentum by stellar winds (Kraft, 1966, 1967).

In the higher luminosity domains of the Hertzsprung-Russell diagram the situation is considerably less clear. In particular, in the case of the stars of highest luminosity, the Ia and Iab supergiants, there is no a priori reason to expect angular momentum to be conserved, for many lines of evidence (Deutsch, 1956; Sargent, 1961; Abt, 1963; Mortón, 1967; Hutchings, 1968) suggest more or less continuous mass loss and hence probable angular momentum loss throughout their evolution.

One can then ask the question whether a study of the change in rotation along an evolutionary track can be used to estimate the changes, if any, in the total angular momentum and from this infer the mass loss rate. The present investigation was undertaken in an attempt to answer this question.

The problem is not straightforward, however, and is complicated by the fact that in these stars the exact nature of the line broadening is not settled. Several good arguments against a strictly rotational interpretation of the line broadening and in favor of broadening due to some form of large-scale mass motion can be given (Huang and Struve, 1953, 1954; Slettebak, 1956; Abt, 1958). In particular, the absence of sharp-lined stars suggests that at least part of the observed line width is due to motion with a spherical rather than an axial form of symmetry.

Therefore the objectives of the present study have been (1) to determine the nature of the line broadening, (2) to see if broadening due to two macroscopic broadening agents (namely axial rotation and large scale turbulence) can be separated, and (3) if the separation is possible, to use the recovered rotational component to study the angular momentum problem. 


\section{The Observational Program}

The observational data consisted of coudé spectrograms of $64 \mathrm{~B}$ and A type Ia and lab supergiants which were obtained with the 84-inch reflector of the Kitt Peak National Observatory. All stars were observed in the blue region at a dispersion of $13.5 \AA / \mathrm{mm}$. Approximately $90 \%$ of all known supergiants observable in the Northern Hemisphere down to a limiting magnitude of $B=8.0$ were observed.

A summary of the observational program is shown in Figure 1. A detailed list of the program stars is contained in Rosendhal (1970). Both field stars and cluster members were observed. We note that the B2-A0 stars are roughly evenly distributed between the two groups whereas the earliest spectral types are predominantly from the general field and the later $\mathrm{A}$ stars are almost all cluster members.

It was our original intent to observe only stars which were members of clusters with known distance moduli. In principle each has a known absolute magnitude and an evolutionary track can be associated with each star in order to determine its main sequence predecessor and to estimate the change in radius and moment of inertia which it has undergone during evolution (cf., Sandage, 1955; Abt, 1957, 1958). However, it was felt that there were not enough objects in this group for a meaningful statistical discussion and so the list was supplemented by a selection of field stars.

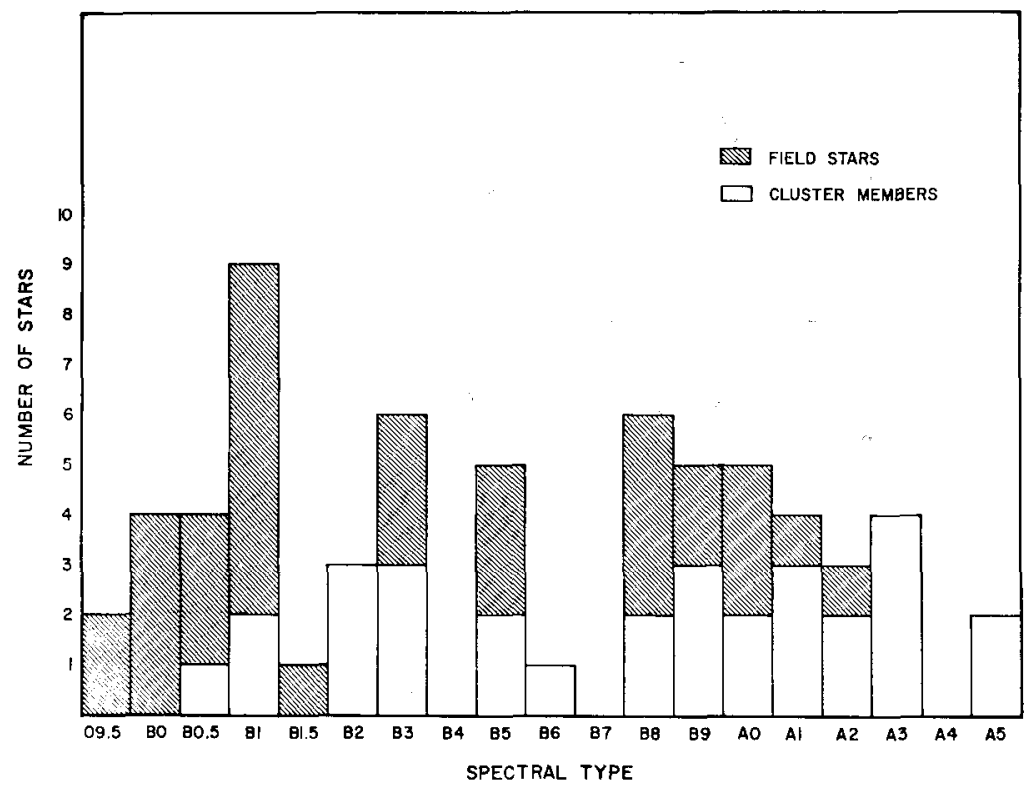

Fig. 1. Distribution of stars in the observational program. There is an even distribution with spectral type and the total sample is almost evenly divided between field stars and cluster members.

\section{Determination of Rotational Velocities}

The rotational velocities were obtained from a comparison of the observed line profiles 
with theoretically computed ones. The lines MgII 4481, He I 4471 and Si IV 4089 were the spectral features which were employed for this purpose. The details of the computations, the reasons for the choice of these lines and a comparison of the results for different lines have been discussed by Rosendhal (1968, 1970).

A comparison of the observed and theoretically computed line profiles for several typical cases is shown in Figure 2. In each case the solid line is the raw data, the dashed line is the adopted smoothed profile (drawn before any theoretical profiles had been computed) and the circles are the theoretical points.

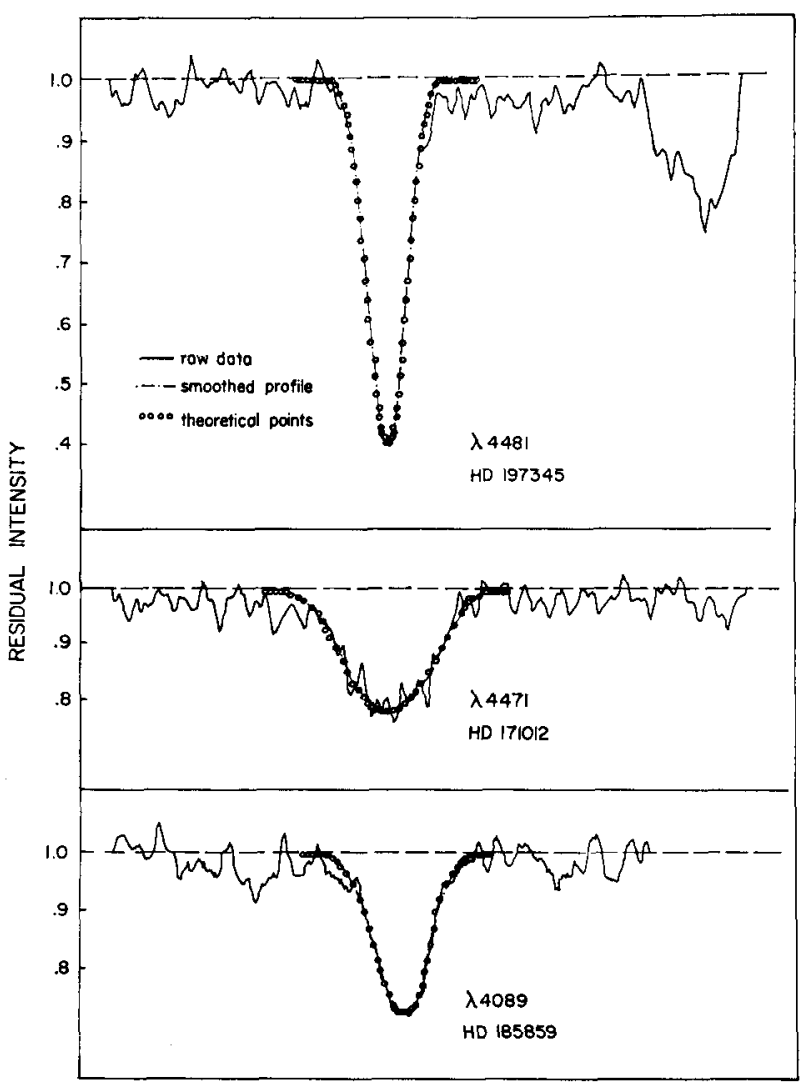

Fig. 2. A comparison of some observed and theoretically computed line profiles (reprinted courtesy of the Astrophysical Journal).

\section{Apparent Rotational Velocities}

The results of the line profile computations are summarized in Figure 3 which shows the behavior of the apparent line broadening expressed as rotation as a function of spectral type. The most important features of this diagram are the absence of any stars showing small apparent rotational velocities, the appreciable spread in broaden- 
ing for the early spectral types, the decreasing spread toward the later spectral types and the relatively smooth decrease exhibited by the lower envelope of the points. The mean apparent rotational velocities for stars in selected small intervals of spectral type are given in the third column of Table I.

\section{TABLE I}

Statistical analysis of supergiant rotation

\begin{tabular}{lclll}
\hline Group & Expansion factor & $\begin{array}{l}\text { Apparent rotation } \\
\left(\mathrm{km} \mathrm{sec}^{-1}\right)\end{array}$ & $\begin{array}{l}\text { Predicted rotation } \\
\left(\mathrm{km} \mathrm{sec}^{-1}\right)\end{array}$ & $\begin{array}{l}\text { Recovered rotation } \\
\left(\mathrm{km} \mathrm{sec}^{-1}\right)\end{array}$ \\
\hline & & & & $13 \pm 6$ \\
A0-A5 & $15-23$ & $\approx 40$ & $8 \pm 4$ & $14 \pm 9$ \\
B3-B6 & $7-10$ & $\approx 60$ & $18 \pm 8$ & $40 \pm 20$ \\
O9.5-B1 & $<5-6$ & $\approx 90$ & $26 \pm 10$ & \\
\hline
\end{tabular}

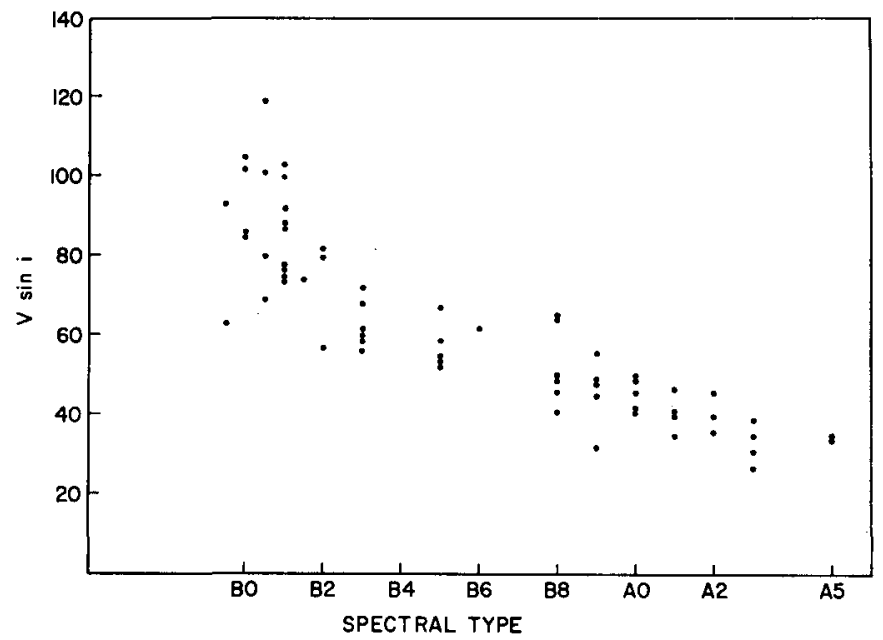

Fig. 3. Line broadening expressed as rotation as a function of spectral type. If two lines have been used in the determination of rotation, the averaged results are shown (reprinted courtesy of the Astrophysical Journal).

\section{Stellar Rotation and Stellar Evolution}

The rotational velocities expected on the basis of evolution away from the main sequence with angular momentum conserved have been calculated with the aid of theoretical evolutionary tracks published by Hayashi and Cameron (1962), Iben (1966) and Stothers (1966). Details of the procedure have been published elsewhere (Rosendhal, 1970).

The two well-known limiting cases (Oke and Greenstein, 1954) of (1) no radial exchange of angular momentum (angular momentum conserved in spherical shells), and (2) free radial exchange of angular momentum (rigid body rotation at all times) 
have been considered. Detailed calculations have been made for case (1). Because of the absence of detailed published information on the density distributions of the appropriate models, only estimates have been made for case (2). In making these estimates, we have used the result (Oke and Greenstein, 1954; Kraft, 1968) that, for a given initial velocity, the final velocity for case (2) always exceeds that for case (1) by about a factor of 2. Recent computations by Faber and Danziger (1969) based on unpublished results of Iben indicate that this factor of two may be incorrect for the A stars. However their computations also indicate that this estimate is reliable for the $B$ stars and it is felt that our basic conclusions will not be affected by these new results.

The expansion factors and rotational velocities expected on the basis of stellar evolution with angular momentum conserved in spherical shells are given in the second and fourth columns of Table I. The error estimates on the predicted velocities reflect uncertainties in effective temperature scales, bolometric corrections and predecessor rotational velocities and have been obtained by propagating possible limiting values through the computations.

\section{Separation of Rotation and Macroturbulence}

In order to separate the contributions to the observed line broadening arising from macroturbulence and axial rotation, we have made use of the fact that turbulence and rotation can be expected to exhibit different symmetries. As many supergiants as possible have been observed and the macroscopic line broadening initially computed as if it were all due to rotation. If results are grouped for stars with similar physical characteristics, in a large enough statistical sample one would expect to see a few stars which are either intrinsic slow rotators or are viewed at small inclination angles. For each group of stars we then attribute the minimum apparent rotation to broadening by large scale turbulence and shift the zero point of the distribution of rotational velocities in order to remove the contribution of large scale turbulence.

In adopting this procedure we have assumed that: (1) in any small box in the Hertzsprung-Russell diagram there is some large scale turbulent velocity which is characteristic of all stars in that box (cf., Meadows, 1961) and (2) the broadening functions for large-scale mass motion and rotation are linearly additive. The validity of these assumptions has been discussed elsewhere (Rosendhal, 1968, 1970). In the actual separation it has been assumed that the observed minimum is due to an aspect effect and a correction has been made (Rosendhal, 1968) to take account of the fact that in a small statistical sample the probability of seeing a star exactly pole-on is very small and that at least part of the observed minimum line broadening may be due to projected rotation.

The recovered rotational component of the observed line broadening for several groups of stars is given in the fifth column of Table I. The error estimates reflect the effect of photometric uncertainties on the observed minimum line broadening and also uncertainties in the geometrical orientation of the sharpest-lined star. 


\section{Discussion}

Consideration of the third, fourth and fifth columns of Table I suggests the following conclusions:

(1) Purely on evolutionary grounds, it appears that axial rotation cannot be the sole source of line broadening in the Ia supergiants. Unless the final velocities from case (2) can be expected to exceed those from case (1) by a factor of 3 or more, the observed broadening expressed as rotation is too high in all cases to be consistent with any possible model of angular momentum conservation. We note that while the recent calculations of Faber and Danziger (1969) may raise some question as to the validity of this statement in the case of the A stars, they do not affect this conclusion for the case of the early B stars. In any event, the absence of sharp lined stars still remains a powerful argument for the presence of some sort of large-scale mass motion in addition to rotation.

(2) At all spectral types in the range B0-A5 both rotation and macroturbulence contribute to the observed line broadening. In particular, in the early B stars rotation is as important a broadening agent as large-scale mass motion. In the middle B and A stars turbulence dominates but there still is an appreciable contribution from rotation.

(3) In spite of the fact that the stars observed seem to be losing mass, we find no strong evidence for significant angular momentum loss. This conclusion is similar to that reached by Abt (1958) in his study of the Ib supergiants. In the later spectral types angular momentum appears to be conserved in spherical shells. Among the early B stars, on the other hand, the fact that the observed rotation is appreciably larger than that predicted from the evolutionary tracks may indicate that these stars are rotating as rigid bodies. We note that the members of this group have not expanded by very large factors. Abt's (1958) suggestion that during initial expansion (to a factor of about 4) a star rotates as a rigid body but that subsequently it commences to rotate differentially may also be true for the stars under consideration in this investigation.

The conclusion that angular momentum appears to be conserved is rather surprising. In fact this investigation was initiated with the expectation that the opposite conclusion would be reached.

We conclude by offering two suggestions to explain this result. The first possibility is that mass loss is not an efficient means of removing angular momentum from these stars. This, in turn, might imply something about the presence or absence of magnetic fields in these stars. In a different context Schatzman $(1959,1962)$ has shown that an outflow of ionized matter in the presence of a magnetic field exerts appreciable torques on a star and can be a very efficient way of braking a star's rotation. A second possibility is that emission and hence mass loss is a recent phenomenon in the history of these stars and the evolutionary changes occur on such a rapid time scale that there has not, as yet, been an opportunity for a significant amount of angular momentum to have been transported away.

A more extensive account of this work has appeared in the January 1970 Astrophysical Journal under the title 'Evolutionary Effects in the Rotation of Supergiants'. 


\section{References}

Abt, H. A.: 1957, Astrophys. J. 126, 503.

Abt, H. A.: 1958, Astrophys. J. 127, 658.

Abt, H. A.: 1963, quoted by R. Weymann in Ann. Rev. Astron. Astrophys. 1, 113.

Deutsch, A. J.: 1956, Astrophys. J. 123, 210.

Faber, S. M. and Danziger, I. J.: 1969, this Volume, p. 39.

Hayashi, C. and Cameron, R. C.: 1962, Astrophys. J. 136, 166.

Huang, S.-S. and Struve, O.: 1953, Astrophys. J. 118, 463.

Huang, S.-S. and Struve, O.: 1954, Ann. Astrophys. 17, 85.

Hutchings, J. B.: 1968, Monthly Notices Roy. Astron. Soc. 141, 329.

Iben, I.: 1966, Astrophys. J. 143, 516.

Kraft, R. P.: 1966, Astrophys. J. 144, 1008.

Kraft, R. P.: 1967, Astrophys. J. 150, 551.

Kraft, R. P.: 1968, in Otto Struve Memorial Volume (ed. by G. H. Herbig), in preparation.

Meadows, A. J.: 1961, Monthly Notices Roy. Astron. Soc. 123, 81.

Morton, D. C.: 1967, Astrophys. J. 150, 535.

Oke, J. B. and Greenstein, J. L.: 1954, Astrophys. J. 120, 384.

Rosendhal, J. D.: 1968, unpublished Ph.D. thesis, Yale University.

Rosendhal, J. D.: 1970, Astrophys. J. 159, 107.

Sandage, A. R.: 1955, Astrophys. J. 122, 263.

Sargent, W. L. W.: 1961, Astrophys. J. 134, 142.

Schatzman, E.: 1959, in I.A.U. Symposium No. 10: The Hertzsprung-Russell Diagram (ed. by J. L. Greenstein), (Ann. Astrophys. Suppl. No.8), p. 129.

Schatzman, E.: 1962, Ann. Astrophys. 25, 18.

Slettebak, A.: 1956, Astrophys. J. 124, 173.

Stothers, R.: 1966, Astrophys. J. 143, 91.

\section{Discussion}

Faber: I would like to point out that your recovered velocities depend in some cases rather strongly on your assumption that turbulent broadening and rotational broadening add linearly. Assuming they add Gaussianly would give considerably higher rotational velocities. From mathematical experiments with test profiles, that is, by rotating profiles already rotated slightly, I find that two forms of macroscopic broadening appear to add much more like the convolution of two Gaussian profiles. If this is true, your subtraction scheme would be a substantial over-correction.

Rosendhal: I agree that there is some question as to the validity of the assumption of linearity. However, I am not sure that your numerical experiments are completely relevant to the problem. What you are doing is changing the unbroadened line profile on each small element of the star's disk and then broadening this new intrinsic line profile. It is not clear to me that this is the same as adding two macroscopic motions. It depends on the relative sizes of the actual macroscopic mass motions and the geometrical elements on the disk and I don't think it is possible to say anything about this. Also, if you look at the group of early B stars for which the factor of two between the evolutionary predictions for rigid body and differential rotation is approximately correct and assume that the broadening adds as the sum of the squares you then find that the recovered rotation is higher than the predicted rotation for both limiting cases. This is obviously unreasonable. Therefore, I think that treating the addition as the convolution of two Gaussians may be a substantial under-correction. The truth probably lies somewhere in between.

Hardorp: What type of line broadening did you use for HeI 4471 ?

Rosendhal: I assumed a Voigt profile, including both thermal and microturbulent Doppler broadening with a small value of the damping constant.

Hardorp: The helium lines are mainly broadened by Stark effect. In your case you may be able to squeeze this into a Voigt profile since the forbidden component is rather less important than on the main sequence. However, a detailed broadening theory for this line has been published by Griem in the Astrophysical Journal, December 1968.

Rosendhal: First, I see no evidence for the presence of the forbidden component on any of my 
plates. Second, at the electron pressures to be expected in supergiant atmospheres (as estimated crudely from counting Balmer lines, for example), unpublished calculations by Peterson indicate that the half-width of the Stark pattern is much smaller than any of the other characteristic half-widths and a negligible error is made by neglecting the Stark effect in this case. In any event, the observed line profiles show no trace of characteristic wings. It should also be remarked that it would seem to make no sense to apply a detailed broadening theory to stellar atmospheres whose structures are as poorly understood as those of the early-type supergiants.

Buscombe: In dealing with such a deep line as $\mathrm{MgII}_{4} 4481$ in A stars, have you taken account of curve-of-growth effects?

Rosendhal: Yes. The microturbulent velocity as obtained from curve-of-growth studies has been included in the intrinsic line profile in order to appropriately unsaturate the line.

Buscombe: Have you evidence of changes with time in the parameters affecting line-broadening?

Rosendhal: Yes. In a paper currently in preparation by Wegner and myself we have examined a time sequence of spectra of several A supergiants and have found that the microturbulence changes with time. We are still examining the data to see if there is any evidence for changes in the macroturbulence.

Buscombe: Was there not a discussion by Steffey (Ph.D. thesis, University of Arizona, 1966) that what you describe as 'field supergiants' have their parentage in associations of young stars?

Rosendhal: Not that I am aware of. In any event, there appear to be no differences in the results which I obtained for field stars and for cluster members and so the parentage of the stars under consideration does not seem to be an important issue.

Bottemiller: On the question of slight variability, one may recall a paper by Serkowski where he reported variable polarization in three early-type supergiants.

Jaschek: Did you look into a correlation between the rotational velocities you measured and the existence of emission lines and/or variability in the spectrum or radial velocity?

Rosendhal: All the plates I took were in the blue and with a few exceptions there were no emission features on my plates. Therefore I can say nothing about such a correlation. I can only comment that the best lines for rotational velocity studies are in the blue and most emission features are in the red region of the spectrum. Several studies including one currently underway by $\mathrm{Abt}$ and myself indicate that the strengths of the emission features in some stars change with time. Therefore in looking for such a correlation you would have to take plates in both regions of the spectrum at approximately the same time. 